EDITORIAL

\section{Alcohol and University Student Drinking - Not a Class Act}

\author{
Wayne Mitic
}

$\mathrm{N}$ o longer adolescents and not yet adults, thousands of undergraduate students enter Canadian colleges and universities each year. While a place for intellectual pursuits and enlightenment, universities are also a social place and, for many students, alcohol is an essential ingredient in their quest for connection and interaction. Gliksman et al. ${ }^{1}$ present the first national study on the prevalence and frequency of heavy drinking (defined as $5+$ and $8+$ drinks per drinking episode) among a nationally representative sample of undergraduate university students. To read their findings as a 1970 s graduate, which I am, is to experience a sense of déjà vu. To read them as a parent of a student currently attending a Canadian university is to experience a foreboding sense of dread. ${ }^{2}$

While it comes as no surprise that most students drink, the timely analysis presents other compelling information. However, as with most research, it also generates further questions for examination:

- Two thirds of students categorized as drinkers consumed at least five drinks per episode in the last year, while one third consumed at least eight drinks per episode. This amount of drinking, usually referred to as binge drinking or high-risk episodic drinking, is a marker for students who will experience subsequent difficulties as a result of their drinking. Some researchers, however, claim that this measure is methodologically flawed since it fails to take into consideration the time over which consumption occurred as well as the size, gender and tolerance of the drinker. ${ }^{3}$ Further research is required on the impact of this consumption pattern, not only on these students, but also on those around them and the surrounding community.

- Heavier drinking is associated with a high recreation and low academic orientation, although one cannot attribute causality solely based on association. Thus, the questions arise: Do people attracted to recreational pursuits tend to be sensation seekers and therefore also drink heavily, or are heavy drinkers simply attracted to these events? Recreation orientation is a broad concept that ranges from actual participation in recreation activities to being a passive observer, and is an area of academic study.

- Little difference in drinking patterns exists across the country except in Quebec where students appear to drink less heavily. The authors suggest that the transition years spent attending CEGEP may influence the reasons why students may drink, however broader cultural issues merit exploration.

- No association was found between heavy drinking and year of study. This finding was not anticipated. One would assume that first-year students, many living away from home for the first time, would "party" more heavily. As well, the negative association between heavy alcohol consumption and academic performance might suggest that these drinkers would fail or drop out of university. This is not the case.

Given these results and the realization that a sizeable percentage of students - both drinkers and others affected by their behaviours - are likely experiencing drinking-related difficulties as a consequence, ${ }^{4}$ what are we to make of this study's findings? First, student alcohol use, and heavy use, occurs at all institutions of higher learning. While consumption rates may vary slightly, this phenomenon is universal. Second, episodic heavy use of alcohol is not unique to university students. It occurs among same-age peers who are not in university, although not to the degree observed within the university population. ${ }^{5}$ Many

Adjunct faculty member, School of Nursing and School of Child and Youth Care, University of Victoria; Research Associate, Institute of Health Promotion Research, UBC

Correspondence: Mr. Wayne Mitic, PO Box 9207 Stn Prov Govt, Victoria, BC V8W 9J1. Tel: 250387-0084; Fax: 250-356-0837; E-mail: wayne.mitic@gems5.gov.bc.ca university students who drink heavily begin this behaviour while still in high school, ${ }^{6}$ and in many cases consumption does decrease once students graduate from university, enter the workforce, marry and start families. ${ }^{7}$ Third, while heavy alcohol use will never be totally eradicated from university campuses, sufficient research exists on why students drink and how these behaviours can be influenced. Results such as those presented by Gliksman et al. provide us with clues as to where our preventive initiatives should take place and whom should be targeted.

\section{Preventive efforts}

Numerous preventive activities have occurred on university campuses over the years to curb the drinking behaviours of students. While some overlap occurs, these activities fall into four broad categories:

Awareness raising: Traditionally, universities have relied upon general awarenessraising campaigns during orientation week, special events and peer education programs as a means of informing students of the risks associated with heavy drinking. ${ }^{8}$ Some faculty have incorporated information on high-risk drinking into their classes, a process called "curriculum infusion", while others have suggested a mandatory, one-credit class for incoming freshmen on how to drink and stay out of trouble. ${ }^{2}$ Awareness-raising initiatives are based on the premise that students lack knowledge of alcohol's effects, of the risks associated with heavy drinking and of the existence of campus policies on responsible use. While it is necessary to provide information, that alone is usually not sufficient in changing individual or collective behaviour. ${ }^{10}$ Most of our preventive efforts, however, still focus on individual behaviour even though most drinking still occurs in group settings where people tend to drink like their peers. ${ }^{11}$ Despite all the educational campaigns, warnings and educational materials, many students still view drinking as a rite of passage and over half drink to get drunk. $^{12}$

Social norms marketing: Research initially conducted by Perkins and Berkowitz ${ }^{13}$ in the 1980s revealed that regardless of the type of drinker or level of use, most drinkers thought that campus drinking norms were greater than personal use. Perkins ${ }^{14}$ suggested that this "reign of error" powerfully influenced student 
drinking behaviours by producing a selffulfilling prophecy - the more students think others drink, the more they themselves will drink. Marketing campaigns conveying accurate information on actual behavioural norms have been successfully initiated and have reduced heavy episodic alcohol consumption and alcohol-related harm. ${ }^{15}$

Environmental change: Decisions students make about alcohol use are shaped by physical, social, economic and legal elements. ${ }^{8}$ Most prevention initiatives on university campuses focus on educating or changing the perceptions of drinkers themselves, providing counselling or short-term treatment, and imposing penalties for the most severe infractions. ${ }^{16}$ However, numerous environmental conditions encourage heavy alcohol use, including ready access to cheap alcohol in high volume, lax or vague alcohol policies, and the culture on some campuses and in some university residences. Several evidencebased strategies aimed at altering environments include: changes in local zoning ordinances to reduce the density of alcohol sales outlets, influencing marketing and sales practices of vendors, changing hours of sales, instituting responsible beverage services, eliminating alcohol-related sponsorship of on-campus activities, and establishing alcohol-free dormitories. ${ }^{17,18}$

Harm reduction activities: Harm reduction initiatives separate the act of drinking from the consequences and focus specifically on reducing the latter. This pragmatic approach acknowledges that using shame, guilt and threats to try and force students to adopt certain behaviours has limited effect. Single ${ }^{19}$ suggests that a first step would be to provide the best information possible on how to decrease the chances of being harmed by drinking. Other strategies include padded furniture in drinking establishments, designated driver programs, and drinking glasses that shatter when broken.

\section{Creating a caring environment}

Research repeatedly demonstrates that a "one-size-fits-all" approach to prevention has limited impact. ${ }^{12}$ Instead, a balance must occur between control measures aimed at restricting drinking levels and harm reduction strategies to reduce the harms associated with heavy use. ${ }^{19}$ To strike such a balance and ascertain key prevention ingredients first requires a broadening of our scope and an investigation of the relationship that exists on university campuses between students and the university administration, and how this relationship is changing.

Since the inception of residential colleges, university authorities have had the power of surrogate parents to make decisions "...concerning the physical and moral welfare and mental training of the pupils." ${ }^{20}$ It was essentially a doctrine under which student affairs personnel promulgated rules (parietals) and provided food, shelter, and discipline, and cared for students if they became ill. ${ }^{11}$ Discipline was enforced through a system of curfews, bed checks, dorm searches, hall monitors and severe repercussions if alcohol was found on the premises, let alone if drunkenness occurred. ${ }^{21}$ While this form of in loco parentis may have met its demise, consensus is lacking on what has replaced it.

Boyer $^{21}$ observed that over the past few decades, universities have gone from being surrogate parents to being clinicians. In the wake of the in loco parentis doctrine "... is a system that eschews connection in the name of student rights, on the one hand, and liability-avoidance on the other."11 What emerged in the aftermath of universityas-parent was a contract or consumer model. Today, students shop for their learning experiences in an educational supermarket. As long as they abide by the rules and regulations concerning student behaviour on campus, universities are eager to treat them like adults. This allows the university to decline responsibility for the consequences that befall students engaged in risky behaviours.

The outcome of this new era of studentuniversity relationship is disconnection. As both university and student self-interest prevail, the sense of community begins to unravel. ${ }^{11}$ As a result, university administrators become unsure of their responsibility, if any, in monitoring and shaping student conduct. Consequently, students emerging from high school, where the message was "thou shalt not", suddenly find themselves in university (and reaching legal drinking age), where the message is...well, either nowhere to be found or phrased in highly punitive, paternalistic terms. One result of this arms-length approach is that some students drink with reckless abandon.

What process exists to move universities from individuals, rules and rights to connection and caring? Key ingredients would include an examination of the values projected by the institutions, and of the missions they embrace, and a better understanding of the expectations students have as they move through this point in their lives. Universities need to examine the complexities of students' lives and the special tensions created by, for example, living in residence, selecting career choices, and relating to others socially. There needs to be a focus on the social context of drinking rather than on the substance itself. Tightening rules would encourage distrust rather than demonstrate care and concern. We should "...encourage what we want, not what we fear." 11

In conclusion, Gliksman et al. draw much-needed attention to the important issue of heavy drinking among Canadian university undergraduate students. The challenge for universities will be to maintain academic excellence and provide students with a safe environment that is conducive to learning, while respecting students' independence and offering them the freedom to develop into responsible and caring adults.

The list of References can be found on page 35. 


\section{L'alcool et les études universitaires : un cocktail qui manque de classe}

\author{
Wayne Mitic
}

Des milliers d'étudiants à mi-chemin entre l'adolescence et l'âge adulte entrent chaque année dans les collèges et les universités du Canada. Lieux de recherche intellectuelle et d'instruction, les universités sont aussi un milieu social, et pour bien des étudiants, l'alcool fait partie intégrante de leur quête d'appartenance et de relations humaines. Gliksman et al. ${ }^{1}$ nous présentent la première étude canadienne sur la prévalence et la fréquence de la consommation abusive d'alcool (qu'ils définissent comme la prise de cinq ou de huit boissons et plus par accès de consommation) au sein d'un échantillon national représentatif d'étudiants d'université du premier cycle. À la lecture de leurs constatations, ceux qui comme moi ont obtenu leur diplôme durant les années 1970 auront une impression de déjà vu, doublée d'une terrible appréhension dans le cas des parents dont la progéniture fréquente actuellement une université canadienne. ${ }^{2}$

Personne ne s'étonnera d'apprendre que la plupart des étudiants consomment de l'alcool, mais l'analyse opportune de Gliksman et al. présente d'autres renseignements probants. Et comme la plupart des études, celle-ci soulève de nouvelles questions :

- Les deux tiers des étudiants dans la catégorie des buveurs avaient pris au moins cinq boissons alcoolisées par accès de consommation au cours de la dernière année, et le tiers en avaient pris au moins huit. Cette consommation excessive, généralement qualifiée d' " alcoolisme périodique ", est un indicateur qui permet de cibler les étudiants qui éprouveront des difficultés en raison de leur consommation d'alcool. Certains chercheurs affirment toutefois que cet indicateur repose sur une méthode imparfaite qui ne tient pas compte du délai dans lequel les boissons sont consommées, ni de la taille, du sexe ou de la tolérance du buveur. ${ }^{3}$ Il faudrait pousser la recherche sur les incidences de l'alcoolisme périodique, non seulement sur les étudiants visés, mais sur leur entourage et dans la collectivité environnante.

- La consommation abusive d'alcool est associée à une prédilection pour les loisirs au détriment des études, mais on ne peut établir de lien causal sur cette seule association. Il y a donc lieu de se demander si les gens attirés par les divertissements ont tendance à être des amateurs de sensations fortes, et donc à boire à l'excès, ou si ce sont les loisirs et les sports qui attirent les buveurs excessifs. La notion de "prédilection pour les loisirs » englobe autant la participation directe aux activités de loisirs et de sports que leur observation passive, et elle fait l'objet d'études théoriques.

- Il existe très peu d'écarts régionaux dans les modes de consommation d'alcool, sauf qu'au Québec, les étudiants semblent faire moins d'excès. Les années de transition passées au cégep ont peut-être une influence bénéfique sur les étudiants, mais les différences culturelles générales entre le Québec et les autres provinces mériteraient d'être examinées.

- On n'a constaté aucun lien entre la consommation abusive d'alcool et l'année d'étude. Ce résultat a de quoi étonner : on s'attendrait à ce que les étudiants de première année, dont beaucoup n'ont jamais quitté le domicile familial auparavant, "fassent la fête " plus souvent que les autres. De plus, l'association négative entre la consommation abusive d'alcool et les résultats scolaires porterait à croire que les buveurs échouent dans leurs études ou les abandonnent. Ce n'est pas le cas.

À la lumière de ces résultats et du fait qu'un pourcentage appréciable d'étudiants - tant les buveurs que ceux qui sont touchés par leur comportement - sont ultérieurement sus-

Membre auxiliaire du corps professoral de l'École des soins infirmiers et de l'École des soins aux enfants et aux adolescents de I'Université de Victoria; agrégé de recherche, Institute of Health Promotion Research, Université de la Colombie-Britannique

Correspondance : M. Wayne Mitic, PO Box 9207 Stn Prov Govt, Victoria (Colombie-Britannique) V8W 9J1. Tél. : (250) 387-0084, téléc. : (250) 356-0837, courriel : wayne.mitic@gems5.gov.bc.ca ceptibles d'éprouver des difficultés liées à l'alcool, ${ }^{4}$ que peut-on penser des constatations de l'étude? Précisons tout d'abord que l'on trouve des buveurs, et des buveurs excessifs, dans la population étudiante de tous les établissements d'enseignement supérieur. Les taux de consommation varient légèrement, mais le phénomène est universel. Deuxièmement, l'alcoolisme périodique n'est pas limité aux étudiants d'université. Ce comportement se retrouve, dans une moindre mesure, chez leurs pairs du même âge qui ne fréquentent pas l'université. ${ }^{5}$ Beaucoup d'étudiants d'université qui boivent à l'excès ont commencé à le faire dès l'école secondaire, ${ }^{6}$ et dans bien des cas, leur consommation diminue lorsqu'ils quittent l'université pour entrer sur le marché du travail, se marier ou fonder une famille. ${ }^{7}$ Troisièmement, bien que la consommation abusive d'alcool ne disparaîtra sans doute jamais complètement des campus universitaires, il existe amplement d'études sur les raisons pour lesquelles les étudiants boivent et sur les moyens d'influencer leur comportement. Les résultats comme ceux de Gliksman et al. nous donnent quelques indices quant aux endroits où mener les initiatives de prévention et aux personnes qu'elles devraient cibler.

\section{Les efforts de prévention}

On mène de nombreuses activités de prévention depuis quelques années, sur les campus universitaires, pour freiner la consommation d'alcool des étudiants. Malgré les chevauchements inévitables, on en distingue quatre grandes catégories :

Les initiatives de sensibilisation : d'habitude, les universités misent sur une grande campagne de sensibilisation durant la semaine d'orientation, sur des activités spécifiques et sur des programmes d'éducation par les pairs pour informer les étudiants des risques associés à la consommation abusive d'alcool. ${ }^{8}$ Certains enseignants intègrent de l'information sur l'excès d'alcool dans leurs cours; ${ }^{9}$ d'autres proposent d'offrir aux étudiants de première année un cours obligatoire d'un crédit pour leur apprendre à boire sans s'attirer d'ennuis. ${ }^{2}$ De telles initiatives supposent que les étudiants connaissent mal les effets de l'alcool, les risques associés à sa consommation abusive et les politiques de consommation responsable en vigueur sur le campus. Il est bien sûr nécessaire de 
fournir de tels renseignements, mais ils ne parviennent pas, à eux seuls, à modifier les comportements individuels et collectifs. ${ }^{10}$ Les efforts de prévention sont encore fortement axés sur les comportements individuels, même si l'alcool se consomme en général dans des lieux de rassemblement où les gens ont tendance à boire comme leurs pairs. ${ }^{11}$ Malgré toutes les campagnes de sensibilisation, les mises en garde et le matériel pédagogique à leur disposition, beaucoup d'étudiants considèrent encore l'alcool comme un rite de passage, et plus de la moitié boivent pour se saoûler. ${ }^{12}$

Les initiatives de promotion des normes sociales: les premières recherches menées par Perkins et Berkowitz ${ }^{13}$ durant les années 1980 ont montré que la plupart des buveurs, peu importe leur catégorie ou leur niveau de consommation, pensent que les niveaux de consommation sur le campus sont supérieurs à leur limite personnelle. Selon Perkins, ${ }^{14}$ ce "règne de l'erreur " influence puissamment la consommation des étudiants, la prophétie finissant par s'accomplir : plus il y a d'étudiants qui pensent que les autres boivent, plus ils ont tendance à boire. On a donc lancé des campagnes pour diffuser des données exactes sur la consommation d'alcool, ce qui a réussi à réduire l'alcoolisme périodique et ses méfaits. ${ }^{15}$

Les initiatives de modification du milieu : lorsqu'un étudiant décide de boire ou de ne pas boire, il est influencé par des facteurs matériels, sociaux, économiques et juridiques. ${ }^{8}$ La plupart des initiatives de prévention sur les campus universitaires visent à sensibiliser les buveurs ou à changer leurs perceptions, à leur offrir du counselling ou des traitements à court terme et à pénaliser les fautes les plus graves. ${ }^{16}$ Toutefois, de nombreuses conditions ambiantes encouragent la consommation abusive d'alcool, notamment l'accès facile aux boissons alcoolisées à bas prix et en grande quantité, le laxisme ou l'imprécision des politiques sur l'alcool et, parfois, la culture des campus et des résidences universitaires. Entre autres stratégies de modification du milieu fondées sur l'expérience clinique, on peut changer les ordonnances de zonage locales pour réduire la densité des points de vente d'alcool, influencer les pratiques de marketing des fournisseurs, modifier les heures de vente, instaurer des services de restauration responsables, inter- dire les commandites liées à l'alcool pour les activités sur le campus ou créer des dortoirs sans alcool. ${ }^{17,18}$

Les initiatives de réduction des méfaits: elles font la distinction entre l'acte de boire et ses conséquences en mettant l'accent sur la réduction des méfaits. Cette approche pragmatique reconnaît que le recours à la honte, à la culpabilité et aux menaces pour forcer les étudiants à adopter certains comportements a peu d'effet. Selon Single, ${ }^{19}$ il faudrait en premier lieu donner le plus d'information possible sur les moyens de réduire les risques liés à l'alcool. D'autres stratégies consistent à installer des meubles rembourrés dans les débits de boissons, à instaurer des programmes de " conducteur désigné " et à fournir des verres qui se fracassent lorsqu'on les brise.

\section{Créer un milieu bienveillant}

La recherche a prouvé à maintes reprises qu'en matière de prévention, les approches générales donnent peu de résultats. ${ }^{12}$ Il est préférable de pondérer les mesures de contrôle de la consommation par des stratégies de réduction des méfaits visant à réduire les préjudices associés à l'abus d'alcool. ${ }^{19}$ Pour atteindre ce juste équilibre et établir avec précision les ingrédients clés de la prévention, il faut d'abord élargir notre champ d'action et étudier la nature et l'évolution de la relation entre les étudiants et l'administration sur les campus universitaires.

Lorsque les centres résidentiels ont fait leur apparition, les autorités universitaires étaient considérées comme des parents de substitution dotés d'un pouvoir décisionnel sur « le bien-être physique et moral et la formation intellectuelle de leurs élèves. " ${ }^{20}$ Selon cette doctrine, les responsables des affaires étudiantes établissaient un règlement (p. ex., sur les heures de visite dans les chambres), procuraient un toit, de la nourriture et des règles disciplinaires aux étudiants et prenaient soin d'eux lorsqu'ils tombaient malades. ${ }^{11}$ On faisait régner la discipline en instaurant des heures de rentrée, en inspectant les lits et les dortoirs et en employant des surveillants de réfectoire. La présence d'alcool sur les lieux, et à plus forte raison l'ivresse, entraînaient de graves répercussions pour les fautifs. ${ }^{21}$ L'époque où les autorités universitaires jouaient le rôle de parents est révolue, mais on ne s'entend pas sur ce qui l'a remplacée.
Boyer $^{21}$ a observé qu'au cours des dernières décennies, les universités sont devenues des cliniciens plutôt que des parents de substitution. On trouve aujourd'hui "...un système qui évacue les relations humaines, au nom des droits des étudiants d'une part et pour limiter la responsabilité de l'université d'autre part. " ${ }^{11}$ L'autorité parentale dévolue aux universités a cédé la place à une relation contractuelle avec les étudiants. Aujourd'hui, ceux-ci "magasinent " leur expérience d'apprentissage dans un supermarché de l'éducation. L'université s'empresse de les traiter en adultes tant qu'ils respectent les règlements du campus, car ainsi, elle n'est pas responsable de ce qui advient à ceux qui adoptent des comportements dangereux.

Il y a de nos jours une rupture de la communication entre l'étudiant et l'université, et elle résulte de cette évolution de leur relation. L'intérêt personnel prime (tant celui de l'université que de l'étudiant), et la notion de bien commun commence à s'effriter. ${ }^{11}$ Par conséquent, les administrateurs des universités ne savent plus très bien jusqu'où s'étend leur responsabilité, si même elle existe, pour ce qui est de surveiller et d'influencer la conduite des étudiants. Et les étudiants frais émoulus de l'école secondaire, avec toutes ses interdictions, se retrouvent soudain à l'université (au moment même où ils atteignent l'âge légal pour boire) et constatent que les interdictions ont disparu ou sont couchées en des termes extrêmement punitifs et paternalistes. Or, en favorisant l'autonomie aux dépens de la responsabilité, on encourage certains étudiants à boire avec une dangereuse insouciance.

Par quels moyens les universités pourraient-elles s'écarter de l'individualisme, des règles et des droits au profit de relations humaines et bienveillantes? Elles devront nécessairement se pencher sur les valeurs qu'elles diffusent et sur la mission qu'elles se sont donnée et essayer de mieux comprendre les attentes que nourrissent les étudiants à cette étape de leur vie. Les universités doivent tenir compte de toutes les facettes de la vie de leurs étudiants et des tensions particulières que suscitent, entre autres, la vie en résidence, les choix de carrière à poser et les relations sociales. Plutôt que de particulariser l'alcool, il faut ... suite à la page 35 
11. Sweanor DT. Tobacco Taxes and Prices in Canada. Ottawa, ON: Smoking and Health Action Foundation, 1997.

12. Adlaf EM, Paglia A. Drug Use Among Ontario Students 1977-2001: Findings from the OSDUS. Toronto, ON: Centre for Addiction and Mental Health, Addiction Research Foundation Division, 2001.

13. Trochim WM. Research Design for Program Evaluation: The Regression Discontinuity Approach. Beverly Hills: Sage Publications, 1994.

14. Stata Corporation. Stata Reference Manual: Release 7. College Station: Stata Corporation, 2000.

15. Hobbs FM, Pickett W, Ferrence R, Brown KS, Madill C, Adlaf EM. Youth smoking in Ontario, 1981-1997: A cause for concern. Can J Public Health 1999;90(2):80-82.

16. Adlaf EM, Ivis FJ. Recent findings from the Ontario Student Drug Use Survey. CMAJ 1998;159:451-54.

17. Ferrence R, Garcia JM, Sykora K, Collishaw NE, Farinon L. Effects of Pricing on Cigarette Use Among Teenagers and Adults in Canada 19801989. Toronto, ON: Addiction Research Foundation, 1991.

18. Sweanor DT. Excise taxes and preventing tobacco use in young people. World Smoking and Health 1992;17:9-12.

19. Stephens T. Workshop report: Trends in the prevalence of smoking, 1991-1994. Chron Dis Can 1995;16:27-32.
20. Mummery WK, Hagen LC. Tobacco pricing, taxation, consumption and revenue: Alberta, 1985-1995. Can J Public Health 1996;87(5):31416.

21. Stephens T, Pederson LL, Koval JJ, Kim C. The relationship of cigarette prices and no-smoking bylaws to the prevalence of smoking in Canada. Am J Public Health 1997;87:1519-21.

22. Hamilton VH, Levinton C, St. Pierre Y, Grimard F. The effect of tobacco tax cuts on cigarette smoking in Canada. CMAJ 1997;156:187-91.
23. Lanoie P, Leclair P. Taxation or regulation: Looking for a good anti-smoking policy. Economics Letters 1998;58:85-89.

24. Harris JE, Chan SW. The continuum-ofaddiction: Cigarette smoking in relation to price among Americans aged 15-29. Health Economics 1999;8:81-86.

Received: August 23, 2001

Accepted: June 19, 2002

\section{RÉSUME}

Contexte : Les jeunes sont particulièrement vulnérables aux fluctuations du prix des cigarettes; or, l'augmentation de la contrebande au début des années 1990 et la réduction des taxes décrétée en 1994 ont facilité l'achat des cigarettes par les jeunes. On examine ici le lien entre ces baisses de prix et les tendances de la prévalence du tabagisme et du nombre de cigarettes fumées par les jeunes en Ontario.

Méthode : On a analysé les données du Sondage sur la consommation de drogues parmi les élèves de I'Ontario pour y déceler des tendances. On a procédé 1) par régression polynomiale et 2) par analyse de discontinuité de la régression pour l'année 1993 afin d'évaluer les effets de la contrebande avant la réduction de taxes, puis les effets de la réduction.

Résultats : Dans l'ensemble, la prévalence du tabagisme a diminué entre 1977 et 1993, est montée en flèche en 1993 et a baissé après 1993. Chez les fumeurs quotidiens, le nombre moyen de cigarettes fumées quotidiennement a augmenté, puis diminué au cours de la période de 24 ans et présente une tendance quadratique négative. Les tendances par sous-groupe sont également indiquées.

Conclusions : D'après ces constatations, la baisse du prix des cigarettes au début des années 1990 pourrait avoir joué un rôle dans l'augmentation du tabagisme des jeunes en Ontario.

\section{Éditorial, suite de la page 16}

s'intéresser au contexte social de sa consommation. En resserrant les règles, on ne fait qu'encourager la méfiance au lieu de manifester sa bienveillance et son souci des étudiants. Il faut "...encourager ce que nous voulons et non ce que nous craignons. ${ }^{11}$

Pour revenir à Gliksman et al., leur étude a le mérite de braquer les projecteurs sur l'important problème de la consommation abusive d'alcool chez les étudiants de premier cycle des universités canadiennes. La difficulté, pour les universités, sera de maintenir l'excellence de l'instruction et de procurer aux étudiants un milieu sûr et propice à l'apprentissage tout en respectant leur autonomie et en leur offrant la liberté de devenir des adultes responsables et compatissants.

\section{RÉFÉRENCES/REFERENCES}

1. Gliksman L, Adlaf E, Demers A, Newton-Taylor B. Heavy drinking on Canadian campuses. Can J Public Health 2003;94(1):17-21.

2. Ruth D. Drinking study puts a spin on higher education. The Tampa Tribune, 2002.

3. See, e.g., Garr M. Comments from the field on the term "Binge Drinking". Higher Education Centre,
2001. Available at http://www.edc.org/hec/comments.html.

4. Wechsler H, Moeykens B, Davenport A, Castillo $S$, Hansen J. The adverse impact of heavy episodic drinkers on other college students. J Stud Alcohol 1995;56:628-34.

5. O'Malley P, Johnston L. Epidemiology of alcohol and other drug use among American college students. J Stud Alc 2002;14:23-39.

6. Wechsler H, Dowdall BW, Davenport A, Castillo S. Correlates of college student drinking. Am J Public Health 1995;85:921-26.

7. Sher K, Bartholow B, Nanda S. Short- and longterm effects of fraternity and sorority membership on heavy drinking: A social norms perspective. Psychol Addict Behav 2001;15( 1):42-51.

8. Presidents Leadership Group. Be vocal, be visible, be visionary: Recommendations for College and University Presidents on Alcohol and Other Drug Prevention. Higher Education Center, 1997. Available at http://www2.edc.org/hec/pubs/.

9. Ryan B, DeJong W. Making the link: Academics and prevention. Higher Education Center, 1998. Available at http://www2.edc.org/hec/pubs/ default.asp.

10. Green LW, Kreuter MW. Health Promotion Planning: An Educational and Environmental Approach. Toronto: Mayfield Publishing Company, 1991.

11. Burns WD, Klawunn M. The web of caring: An approach to accountability in alcohol policy. Higher Education Centre, 1998. Available at http://www. edc.org/hec/pubs/theorybook/burns.html.

12. Wechsler H, Lee J, Kuo M, Seibring M, Nelson $\mathrm{T}$, Lee H. Trends in college binge drinking during a period of increased prevention efforts. $J \mathrm{Am}$ Coll Health 2002;50(5):203-17.
13. Perkins H, Berkowitz A. Perceiving the community norms of alcohol use among students: Some research implications for campus alcohol education programming. Int J Addict 1996;21:961-76.

14. Perkins $\mathrm{H}$. Confronting misperceptions of peer drug use norms among college students. Peer Prevention Program Implementation Manual. Higher Education Leaders/Peers Network, Texas Christian University, 1991.

15. Glider P, Midyett S, Mills-Novoa B, Johannessen $\mathrm{K}$, Collins $\mathrm{C}$. Challenging the collegiate rite of passage: A campus-wide social marketing media campaign to reduce binge drinking. J Drug Educ2001;31(2):207-20.

16. Wechsler H, Kelly K, Weitzman E, SanGiovanni J, Seibring M. What colleges are doing about binge drinking: A survey of college presidents. J Am Coll Health 2000;48:219-26.

17. Preventing problems related to alcohol availability: Environmental approaches. Substance Abuse and Mental Health Services Administration, 2001. Publication no. (SMA)99-3298. Available at http://www.health.org/govpubs/PHD822/ aar.htm.

18. Task Force of the National Advisory Council on Alcohol Abuse and Alcoholism. A Call to Action: Changing the Culture of Drinking at U.S. Colleges. National Institute on Alcohol and Alcoholism, April 2002. Available at http://www.collegedrinkingprevention.gov/ Reports/TaskForce/TaskForce_TOC.aspx

19. Single E. Alcohol and youth: Time for effective action. Can J Public Health 2002;93(3):169-70.

20. Gott v. Berea College. 1913, 156 Ky.376, 161 S.W. 204.

21. Boyer E. College: The Undergraduate Experience in America. New York: Harper \& Row, 1987. 